International Journal of Computer Science \& Information Technology (IJCSIT) Vol 3, No 4, August 2011

\title{
ACtive Controller Design for Global CHAOS ANTI-SYNCHRONIZATION OF LI AND Tigan Chaotic Systems
}

\author{
Sundarapandian Vaidyanathan $^{1}$ and Karthikeyan Rajagopal ${ }^{2}$ \\ ${ }^{1}$ Research and Development Centre, Vel Tech Dr. RR \& Dr. SR Technical University \\ Avadi, Chennai-600 062, Tamil Nadu, INDIA \\ sundarvtuegmail.com \\ ${ }^{2}$ School of Electronics and Electrical Engineering, Singhania University \\ Dist. Jhunjhunu, Rajasthan-333 515, INDIA \\ rkarthiekeyan@gmail.com
}

\begin{abstract}
This paper discusses the design of active controllers for achieving global chaos anti-synchronization of identical Li systems (2009), Tigan systems (2008) and non-identical Li and Tigan systems. Lyapunov stability theory has been deployed for establishing the anti-synchronization results derived in this paper for Li and Tigan chaotic systems. Since the Lyapunov exponents are not required for these calculations, the active nonlinear control method is very effective and suitable to achieve anti-synchronization of identical and non-identical Li and Tigan chaotic systems. Numerical simulations have been presented to illustrate the anti-synchronization results for the chaotic systems addressed in this paper.
\end{abstract}

\section{KEYWORDS}

Active Control, Anti-Synchronization, Chaotic Systems, Li System, Tigan System, Nonlinear Control.

\section{INTRODUCTION}

Chaotic systems are nonlinear dynamical systems which are highly sensitive to initial conditions. This sensitivity of chaotic systems is usually called as the butterfly effect [1].

Chaos synchronization problem received great attention in the literature when Pecora and Carroll [2] published their results on chaos synchronization in 1990. From then on, chaos synchronization has been extensively and intensively studied in the last three decades [3-25]. Chaos theory has been explored in a variety of fields including physical systems [3], chemical systems [4], ecological systems [5], secure communications [6-7], etc.

Synchronization of chaotic systems is a phenomenon that may occur when a chaotic oscillator drives another chaotic oscillator. Because of the butterfly effect which causes the exponential divergence of the trajectories of two identical chaotic systems started with nearly the same initial conditions, synchronizing two chaotic systems is seemingly a very challenging problem.

In most of the chaos synchronization approaches, the master-slave or drive-response formalism is used. If a particular chaotic system is called the master or drive system and another chaotic system is called the slave or response system, then the idea of anti-synchronization is to use the output of the master system to control the slave system so that the states of the slave system have the same amplitude but opposite signs as the states of the master system asymptotically. In other words, the sum of the states of the master and slave systems are designed to converge to zero asymptotically, when anti-synchronization appears.

DOI : $10.5121 /$ ijcsit.2011.3420 
In the recent years, various schemes have been deployed for chaos synchronization such as PC method [2], OGY method [8], active control [9-12], adaptive control [13-15], backstepping design [16], sampled-data feedback [17], sliding mode control [18-20], etc. Recently, active control method has been applied to achieve anti-synchronization of two identical chaotic systems [21-22].

In this paper, we use active control to derive new results for the global chaos antisynchronization of identical Li systems ([23], 2009), identical Tigan systems ([24], 2008) and non-identical Li and Tigan systems.

This paper is organized as follows. In Section 2, we describe the problem statement and our methodology using Lyapunov stability theory. In Section 3, we give a description of the chaotic systems addressed in this paper, viz. Li system (2009) and Tigan system (2008). In Section 4, we derive results for the anti-synchronization of identical $\mathrm{Li}$ systems (2009) using active nonlinear control. In Section 5, we derive results for the anti-synchronization of identical Tigan systems (2008) using active nonlinear control. In Section 6, we derive results for the antisynchronization of $\mathrm{Li}$ and Tigan systems using active nonlinear control. In Section 7, we summarize the main results obtained in this paper.

\section{Problem Statement and Our Methodology}

As the master or drive system, we consider the chaotic system described by

$$
\dot{x}=A x+f(x),
$$

where $x \in R^{n}$ is the state vector, $A$ is the $n \times n$ matrix of system parameters and $f: R^{n} \rightarrow R^{n}$ is the nonlinear part of the system.

As the slave or response system, we consider the following chaotic system described by

$$
\dot{y}=B y+g(y)+u,
$$

where $y \in R^{n}$ is the state of the slave system, $B$ is the $n \times n$ matrix of system parameters, $g: R^{n} \rightarrow R^{n}$ is the nonlinear part of the system and $u$ is the active controller to be designed.

If $A=B$ and $f=g$, then $x$ and $y$ are the states of two identical chaotic systems. If $A \neq B$ or $f \neq g$, then $x$ and $y$ are the states of two different chaotic systems.

For the anti-synchronization of the chaotic systems (1) and (2) using active control, we design a state feedback controller $u$, which anti-synchronizes the states of the master system (1) and the slave system (2) for all initial conditions $x(0), y(0) \in R^{n}$.

If we define the anti-synchronization error as

$$
e=y+x
$$

then the error dynamics is obtained as

$$
\dot{e}=B y+A x+g(y)+f(x)+u
$$


International Journal of Computer Science \& Information Technology (IJCSIT) Vol 3, No 4, August 2011

Thus, the global anti-synchronization problem is essentially to find a feedback controller (active controller) $u$ so as to stabilize the error dynamics (4) for all initial conditions, i.e.

$$
\lim _{t \rightarrow \infty}\|e(t)\|=0, \text { for all } e(0) \in R^{n}
$$

We use the Lyapunov stability theory as our methodology. We take as a candidate Lyapunov function

$$
V(e)=e^{T} P e,
$$

where $P$ is a positive definite matrix. Note that $V: R^{n} \rightarrow R$ is a positive definite function by construction.

If we find a feedback controller $u$ so that

$$
\dot{V}(e)=-e^{T} Q e,
$$

where $Q$ is a positive definite matrix, then $\dot{V}: R^{n} \rightarrow R$ is a negative definite function.

Thus, by Lyapunov stability theory [25], the error dynamics (4) is globally exponentially stable.

\section{SYSTEMS DESCRIPTION}

The Li system ([23], 2009) is described by the dynamics

$$
\begin{aligned}
& \dot{x}_{1}=a\left(x_{2}-x_{1}\right) \\
& \dot{x}_{2}=x_{1} x_{3}-x_{2} \\
& \dot{x}_{3}=b-x_{1} x_{2}-c x_{3}
\end{aligned}
$$

where $x_{1}, x_{2}, x_{3}$ are the state variables and $a, b, c$ are constant, positive parameters of the system.

The Li dynamics (8) is chaotic when the parameter values are taken as $a=5, b=16$ and $c=1$. Figure 1 describes the state portrait of the Li system (8).

The Tigan system ([24], 2008) is described by the dynamics

$$
\begin{aligned}
& \dot{x}_{1}=\alpha\left(x_{2}-x_{1}\right) \\
& \dot{x}_{2}=(\gamma-\alpha) x_{1}-\alpha x_{1} x_{3} \\
& \dot{x}_{3}=-\beta x_{3}+x_{1} x_{2}
\end{aligned}
$$

where $x_{1}, x_{2}, x_{3}$ are the state variables and $\alpha, \beta, \gamma$ are constant, positive parameters of the system.

The Tigan dynamics (9) is chaotic when the parameter values are taken as $\alpha=2.1, \beta=0.6$ and $\gamma=30$. Figure 2 describes the state portrait of the Tigan system (9). 
International Journal of Computer Science \& Information Technology (IJCSIT) Vol 3, No 4, August 2011

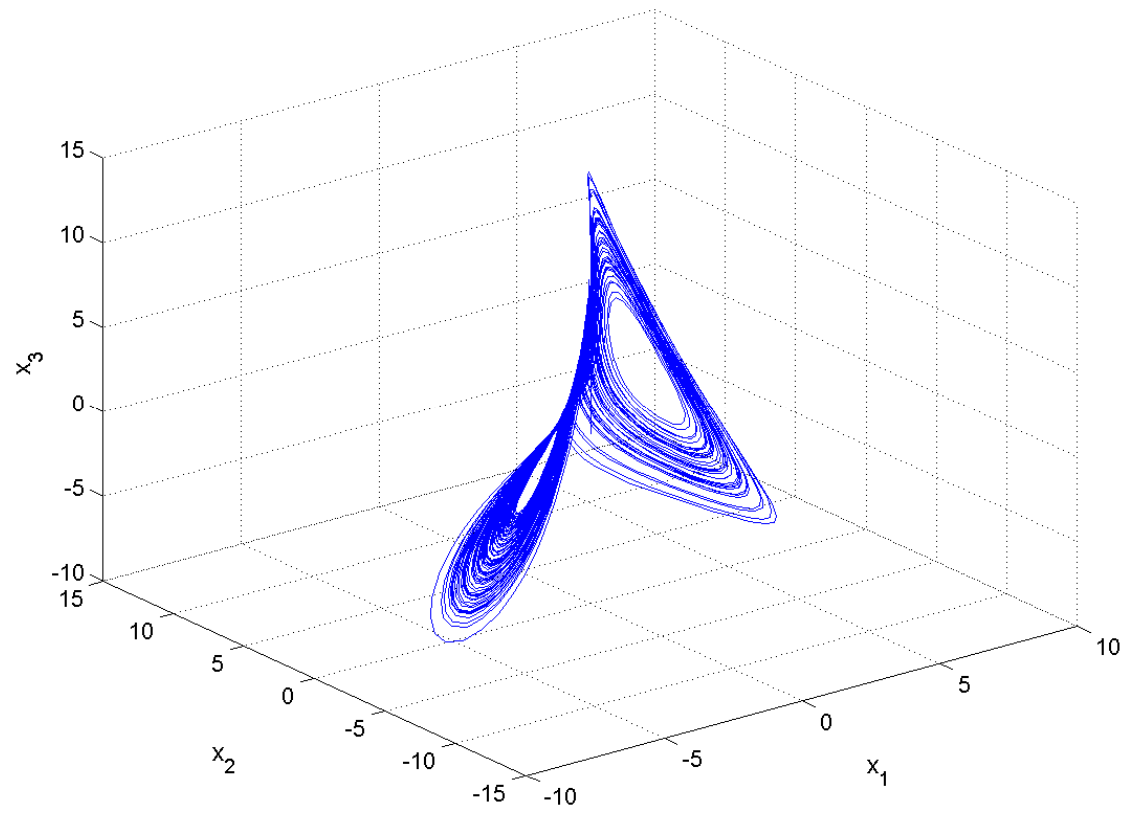

Figure 1. State Orbits of the Li System

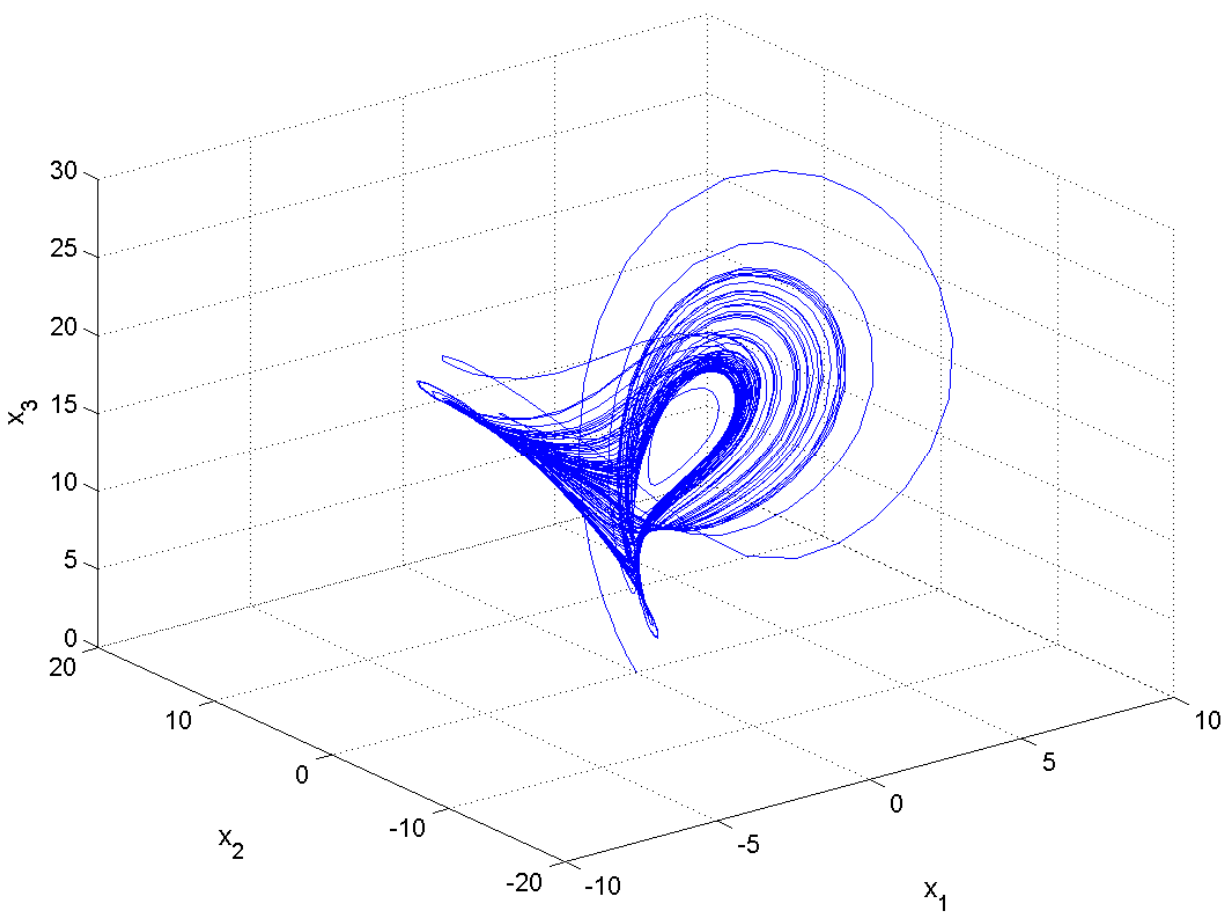

Figure 2. State Orbits of the Tigan System 
International Journal of Computer Science \& Information Technology (IJCSIT) Vol 3, No 4, August 2011

\section{ANTI-SYNCHRONIZATION OF IDENTICAL Li SYSTEMS}

In this section, we consider the anti-synchronization of identical Li systems ([23], 2009).

As the master system, we consider the Li dynamics described by

$$
\begin{aligned}
& \dot{x}_{1}=a\left(x_{2}-x_{1}\right) \\
& \dot{x}_{2}=x_{1} x_{3}-x_{2} \\
& \dot{x}_{3}=b-x_{1} x_{2}-c x_{3}
\end{aligned}
$$

where $x_{1}, x_{2}, x_{3}$ are the state variables and $a, b, c$ are positive constants.

As the slave system, we consider the controlled Li dynamics described by

$$
\begin{aligned}
& \dot{y}_{1}=a\left(y_{2}-y_{1}\right)+u_{1} \\
& \dot{y}_{2}=y_{1} y_{3}-y_{2}+u_{2} \\
& \dot{y}_{3}=b-y_{1} y_{2}-c y_{3}+u_{3}
\end{aligned}
$$

where $y_{1}, y_{2}, y_{3}$ are the state variables and $u_{1}, u_{2}, u_{3}$ are the active controls.

The anti-synchronization error is defined as

$$
\begin{aligned}
& e_{1}=y_{1}+x_{1} \\
& e_{2}=y_{2}+x_{2} \\
& e_{3}=y_{3}+x_{3}
\end{aligned}
$$

A simple calculation gives the error dynamics

$$
\begin{aligned}
& \dot{e}_{1}=a\left(e_{2}-e_{1}\right)+u_{1} \\
& \dot{e}_{2}=-e_{2}+y_{1} y_{3}+x_{1} x_{3}+u_{2} \\
& \dot{e}_{3}=-c e_{3}+2 b-y_{1} y_{2}-x_{1} x_{2}+u_{3}
\end{aligned}
$$

We consider the active nonlinear controller defined by

$$
\begin{aligned}
& u_{1}=-a e_{2} \\
& u_{2}=-y_{1} y_{3}-x_{1} x_{3} \\
& u_{3}=-2 b+y_{1} y_{2}+x_{1} x_{2}
\end{aligned}
$$

Substitution of (14) into (13) yields the linear error dynamics

$$
\begin{aligned}
& \dot{e}_{1}=-a e_{1} \\
& \dot{e}_{2}=-e_{2} \\
& \dot{e}_{3}=-c e_{3}
\end{aligned}
$$


International Journal of Computer Science \& Information Technology (IJCSIT) Vol 3, No 4, August 2011

We consider the quadratic Lyapunov function defined by

$$
V(e)=\frac{1}{2} e^{T} e=\frac{1}{2}\left(e_{1}^{2}+e_{2}^{2}+e_{3}^{2}\right)
$$

which is a positive definite function on $R^{3}$.

Differentiating (16) along the trajectories of the error system (15), we get

$$
\dot{V}(e)=-a e_{1}^{2}-e_{2}^{2}-c e_{3}^{2},
$$

which is a negative definite function on $R^{3}$ since $a$ and $c$ are positive constants.

Thus, by Lyapunov stability theory [25], the error dynamics (15) is globally exponentially stable. Hence, we obtain the following result.

Theorem 1. The identical Li systems (10) and (11) are globally and exponentially antisynchronized with the active nonlinear controller (14).

\section{Numerical Simulations}

For the numerical simulations, the fourth order Runge-Kutta method with initial timestep $h=10^{-6}$ is used to solve the two systems of differential equations (10) and (11) with the nonlinear controller (14).

The Li chaotic system (2009) is one of the important paradigms of three-dimensional chaotic systems.

The parameters of the identical Li systems (10) and (11) are selected as

$$
a=5, b=16, c=1
$$

so that the systems (10) and (11) exhibit chaotic behaviour.

The initial values for the master system (10) are taken as

$$
x_{1}(0)=12, \quad x_{2}(0)=8, \quad x_{3}(0)=25
$$

and the initial values for the slave system (11) are taken as

$$
y_{1}(0)=4, \quad y_{2}(0)=16, \quad y_{3}(0)=26
$$

Figure 3 depicts the anti-synchronization of the identical Li chaotic systems (10) and (11). 
International Journal of Computer Science \& Information Technology (IJCSIT) Vol 3, No 4, August 2011
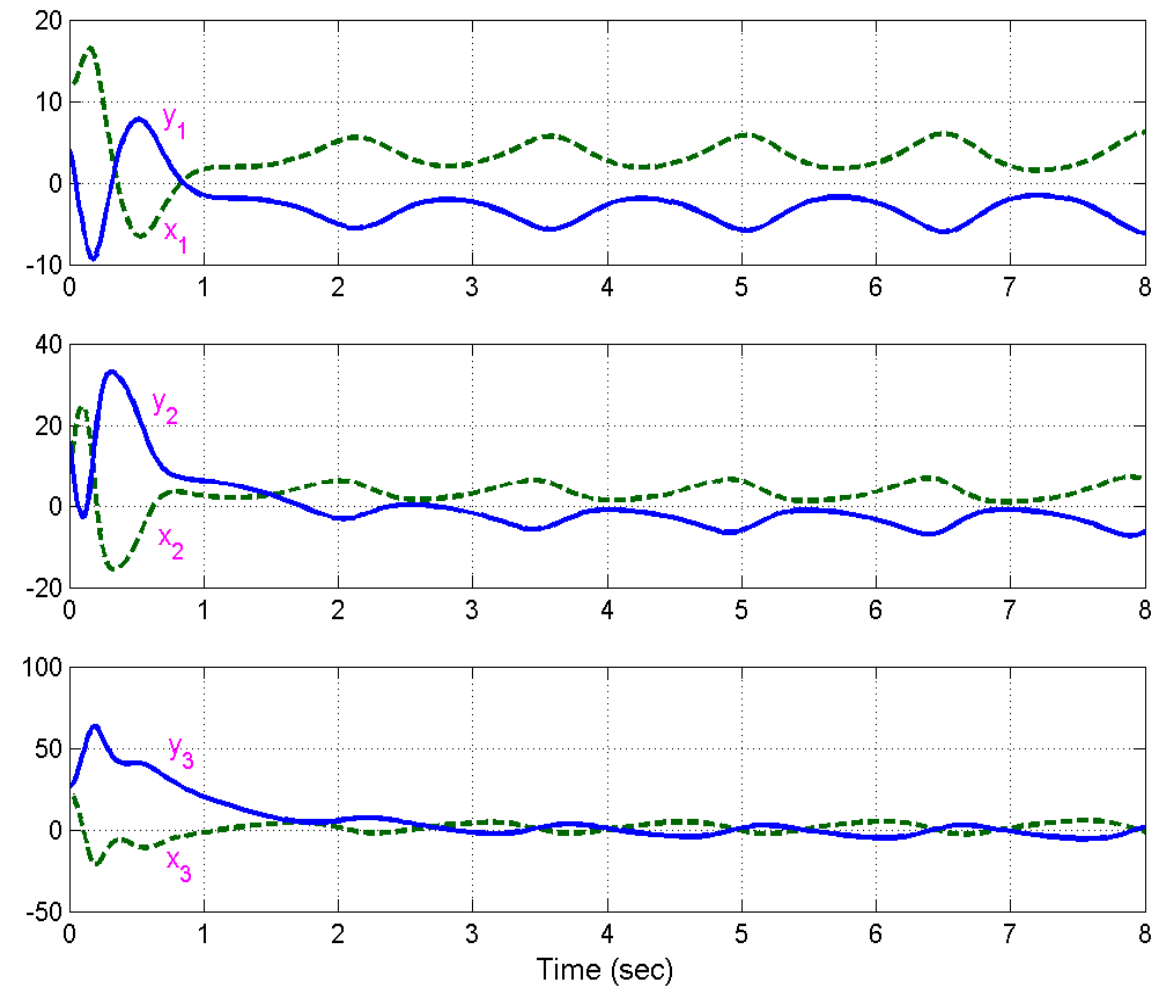

Figure 3. Anti-Synchronization of Identical Li Systems

\section{ANTI-SyNChronization OF IDENTICAL TIGAN SySTEMS}

In this section, we consider the anti-synchronization of identical Tigan systems ([24], 2008).

As the master system, we consider the Tigan dynamics described by

$$
\begin{aligned}
& \dot{x}_{1}=\alpha\left(x_{2}-x_{1}\right) \\
& \dot{x}_{2}=(\gamma-\alpha) x_{1}-\alpha x_{1} x_{3} \\
& \dot{x}_{3}=-\beta x_{3}+x_{1} x_{2}
\end{aligned}
$$

where $x_{1}, x_{2}, x_{3}$ are the state variables and $\alpha, \beta, \gamma$ are positive constants.

As the slave system, we consider the controlled Tigan dynamics described by

$$
\begin{aligned}
& \dot{y}_{1}=\alpha\left(y_{2}-y_{1}\right)+u_{1} \\
& \dot{y}_{2}=(\gamma-\alpha) y_{1}-\alpha y_{1} y_{3}+u_{2} \\
& \dot{y}_{3}=-\beta y_{3}+y_{1} y_{2}+u_{3}
\end{aligned}
$$

where $y_{1}, y_{2}, y_{3}$ are the state variables and $u_{1}, u_{2}, u_{3}$ are the active controls. 
International Journal of Computer Science \& Information Technology (IJCSIT) Vol 3, No 4, August 2011

The anti-synchronization error is defined as

$$
\begin{aligned}
& e_{1}=y_{1}+x_{1} \\
& e_{2}=y_{2}+x_{2} \\
& e_{3}=y_{3}+x_{3}
\end{aligned}
$$

A simple calculation gives the error dynamics

$$
\begin{aligned}
& \dot{e}_{1}=\alpha\left(e_{2}-e_{1}\right)+u_{1} \\
& \dot{e}_{2}=(\gamma-\alpha) e_{1}-\alpha\left(y_{1} y_{3}+x_{1} x_{3}\right)+u_{2} \\
& \dot{e}_{3}=-\beta e_{3}+y_{1} y_{2}+x_{1} x_{2}+u_{3}
\end{aligned}
$$

We consider the active nonlinear controller defined by

$$
\begin{aligned}
& u_{1}=-\alpha e_{2} \\
& u_{2}=-(\gamma-\alpha) e_{1}-e_{2}+\alpha\left(y_{1} y_{3}+x_{1} x_{3}\right) \\
& u_{3}=-\beta e_{3}-y_{1} y_{2}-x_{1} x_{2}
\end{aligned}
$$

Substitution of (22) into (21) yields the linear error dynamics

$$
\begin{aligned}
& \dot{e}_{1}=-\alpha e_{1} \\
& \dot{e}_{2}=-e_{2} \\
& \dot{e}_{3}=-2 \beta e_{3}
\end{aligned}
$$

We consider the quadratic Lyapunov function defined by

$$
V(e)=\frac{1}{2} e^{T} e=\frac{1}{2}\left(e_{1}^{2}+e_{2}^{2}+e_{3}^{2}\right),
$$

which is a positive definite function on $R^{3}$.

Differentiating (24) along the trajectories of the system (23), we get

$$
\dot{V}(e)=-\alpha e_{1}^{2}-e_{2}^{2}-2 \beta e_{3}^{2},
$$

which is a negative definite function on $R^{3}$ since $\alpha$ and $\beta$ are positive constants.

Thus, by Lyapunov stability theory [25], the error dynamics (23) is globally exponentially stable. Hence, we obtain the following result.

Theorem 2. The identical Tigan systems (18) and (19) are globally and exponentially anti-synchronized with the active nonlinear controller (22). 
International Journal of Computer Science \& Information Technology (IJCSIT) Vol 3, No 4, August 2011

\section{Numerical Simulations}

For the numerical simulations, the fourth order Runge-Kutta method with initial timestep $h=10^{-6}$ is used to solve the two systems of differential equations (18) and (19) with the nonlinear controller (22).

The Tigan chaotic system (2008) is one of the important paradigms of threedimensional chaotic systems.

The parameters of the identical Tigan systems (18) and (19) are selected as

$$
\alpha=2.1, \beta=0.6, \gamma=30
$$

The initial values for the master system (18) are taken as

$$
x_{1}(0)=6, \quad x_{2}(0)=17, \quad x_{3}(0)=10
$$

and the initial values for the slave system (19) are taken as

$$
y_{1}(0)=22, \quad y_{2}(0)=30, \quad y_{3}(0)=18
$$

Figure 4 depicts the anti-synchronization of the identical Tigan chaotic systems (18) and (19).
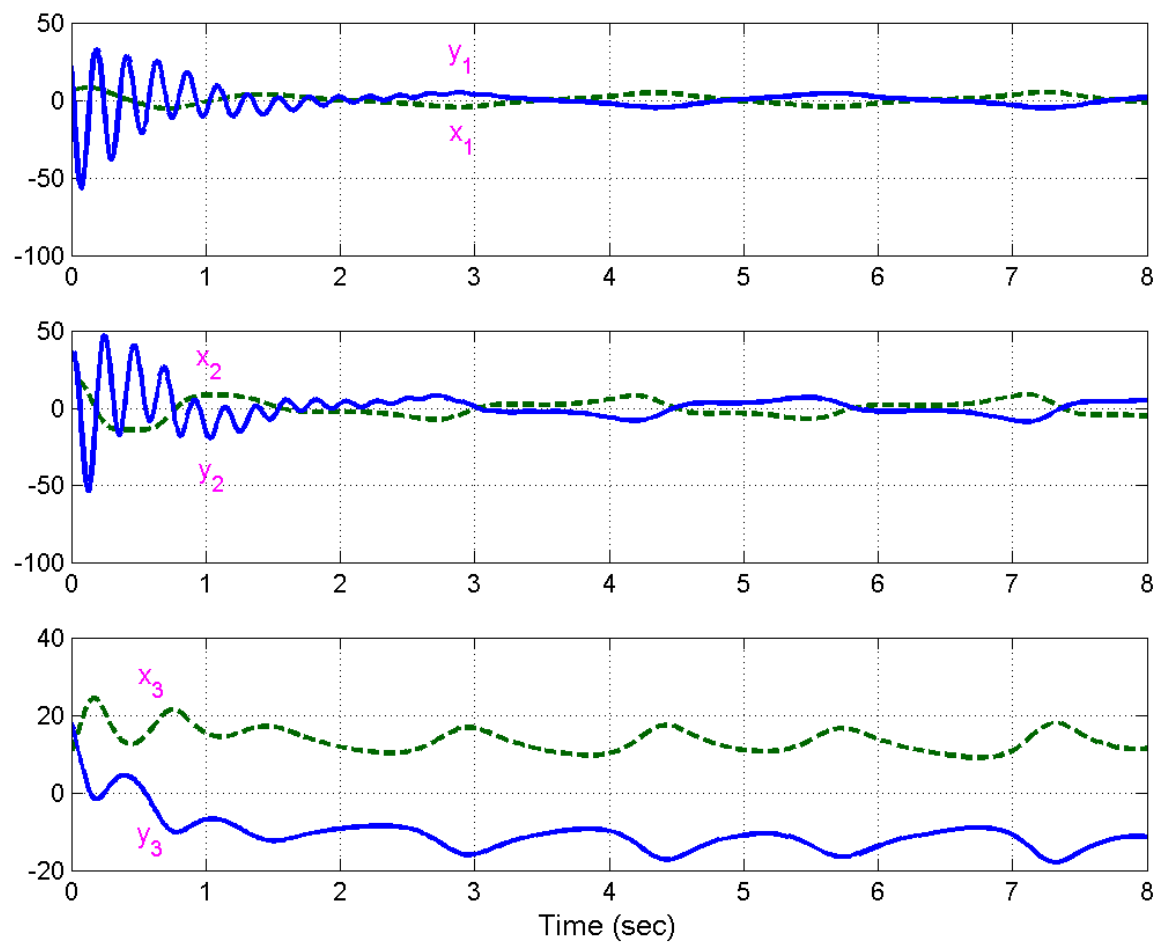

Figure 4. Anti-Synchronization of Identical Tigan Systems 
International Journal of Computer Science \& Information Technology (IJCSIT) Vol 3, No 4, August 2011

\section{ANTI-SYNCHRONIZATION OF LI AND TIGAN SYSTEMS}

In this section, we consider the anti-synchronization of Li and Tigan chaotic systems.

As the master system, we consider the Li dynamics described by

$$
\begin{aligned}
& \dot{x}_{1}=a\left(x_{2}-x_{1}\right) \\
& \dot{x}_{2}=x_{1} x_{3}-x_{2} \\
& \dot{x}_{3}=b-x_{1} x_{2}-c x_{3}
\end{aligned}
$$

where $x_{1}, x_{2}, x_{3}$ are the state variables and $a, b, c$ are positive constants.

As the slave system, we consider the controlled Tigan dynamics described by

$$
\begin{aligned}
& \dot{y}_{1}=\alpha\left(y_{2}-y_{1}\right)+u_{1} \\
& \dot{y}_{2}=(\gamma-\alpha) y_{1}-\alpha y_{1} y_{3}+u_{2} \\
& \dot{y}_{3}=-\beta y_{3}+y_{1} y_{2}+u_{3}
\end{aligned}
$$

where $y_{1}, y_{2}, y_{3}$ are the state variables, $\alpha, \beta, \gamma$ are positive constants and $u_{1}, u_{2}, u_{3}$ are the active controls.

The anti-synchronization error is defined as

$$
\begin{aligned}
& e_{1}=y_{1}+x_{1} \\
& e_{2}=y_{2}+x_{2} \\
& e_{3}=y_{3}+x_{3}
\end{aligned}
$$

A simple calculation gives the error dynamics

$$
\begin{aligned}
& \dot{e}_{1}=\alpha\left(e_{2}-e_{1}\right)+(a-\alpha)\left(x_{2}-x_{1}\right)+u_{1} \\
& \dot{e}_{2}=-e_{2}+(\gamma-\alpha) y_{1}+y_{2}-\alpha y_{1} y_{3}+x_{1} x_{3}+u_{2} \\
& \dot{e}_{3}=-\beta e_{3}+(\beta-c) x_{3}+b+y_{1} y_{2}-x_{1} x_{2}+u_{3}
\end{aligned}
$$

We consider the active nonlinear controller defined by

$$
\begin{aligned}
& u_{1}=-\alpha e_{2}-(a-\alpha)\left(x_{2}-x_{1}\right) \\
& u_{2}=-(\gamma-\alpha) y_{1}-y_{2}+\alpha y_{1} y_{3}-x_{1} x_{3} \\
& u_{3}=-\beta e_{3}-(\beta-c) x_{3}-b-y_{1} y_{2}+x_{1} x_{2}
\end{aligned}
$$

Substitution of (30) into (29) yields the linear error dynamics

$$
\begin{aligned}
& \dot{e}_{1}=-\alpha e_{1} \\
& \dot{e}_{2}=-e_{2} \\
& \dot{e}_{3}=-2 \beta e_{3}
\end{aligned}
$$


International Journal of Computer Science \& Information Technology (IJCSIT) Vol 3, No 4, August 2011

We consider the quadratic Lyapunov function defined by

$$
V(e)=\frac{1}{2} e^{T} e=\frac{1}{2}\left(e_{1}^{2}+e_{2}^{2}+e_{3}^{2}\right)
$$

which is a positive definite function on $R^{3}$.

Differentiating (32) along the trajectories of the system (31), we get

$$
\dot{V}(e)=-\alpha e_{1}^{2}-e_{2}^{2}-2 \beta e_{3}^{2},
$$

which is a negative definite function on $R^{3}$ since $\alpha$ and $\beta$ are positive constants.

Thus, by Lyapunov stability theory [25], the error dynamics (31) is globally exponentially stable. Hence, we obtain the following result.

Theorem 3. The non-identical Li system (26) and Tigan system (27) are globally and exponentially anti-synchronized with the active nonlinear controller (30).

\section{Numerical Simulations}

For the numerical simulations, the fourth order Runge-Kutta method with initial timestep $h=10^{-6}$ is used to solve the two systems of differential equations (26) and (27) with the nonlinear controller (30).

The Li chaotic system (2009) is one of the important paradigms of three-dimensional chaotic systems.

The Tigan chaotic system (2008) is also one of the important paradigms of threedimensional chaotic systems.

The parameters of the Li system (26) are selected as

$$
a=5, b=16, c=1
$$

The parameters of the Tigan system (27) are selected as

$$
\alpha=2.1, \beta=0.6, \gamma=30
$$

The initial values for the master system (26) are taken as

$$
x_{1}(0)=10, \quad x_{2}(0)=25, \quad x_{3}(0)=9
$$

and the initial values for the slave system (27) are taken as

$$
y_{1}(0)=26, \quad y_{2}(0)=4, \quad y_{3}(0)=17
$$

Figure 5 depicts the anti-synchronization of the non-identical Li and Tigan chaotic systems. 

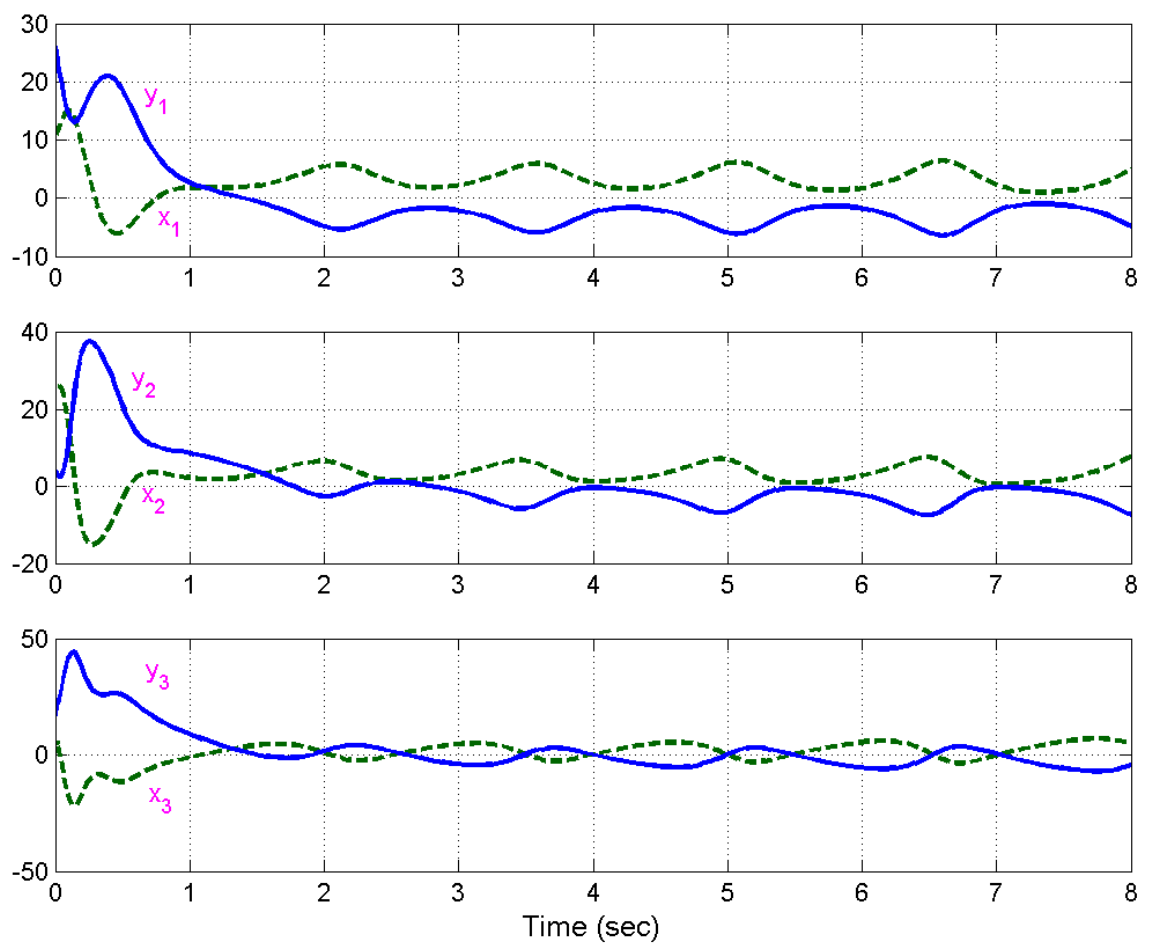

Figure 5. Anti-Synchronization of Li and Tigan Systems

\section{Conclusions}

In this paper, using the active control method, new results have been derived for the antisynchronization for the identical Li systems (2009), identical Tigan systems (2008) and nonidentical Li and Tigan systems. The anti-synchronization results derived in this paper have been established using Lyapunov stability theory. Since the Lyapunov exponents are not required for these calculations, the active control method is very convenient and efficient for the antisynchronization of identical and non-identical $\mathrm{Li}$ and Tigan systems. Numerical simulation results have been presented to illustrate the anti-synchronization results derived in this paper.

\section{REFERENCES}

[1] Alligood, K.T., Sauer, T. \& Yorke, J.A. (1997) Chaos: An Introduction to Dynamical Systems, Springer, New York.

[2] Pecora, L.M. \& Carroll, T.L. (1990) "Synchronization in chaotic systems", Phys. Rev. Lett., Vol. 64, pp 821-824.

[3] Lakshmanan, M. \& Murali, K. (1996) Nonlinear Oscillators: Controlling and Synchronization, World Scientific, Singapore.

[4] Han, S.K., Kerrer, C. \& Kuramoto, Y. (1995) "Dephasing and burstling in coupled neural oscillators", Phys. Rev. Lett., Vol. 75, pp 3190-3193.

[5] Blasius, B., Huppert, A. \& Stone, L. (1999) "Complex dynamics and phase synchronization in spatially extended ecological system”, Nature, Vol. 399, pp 354-359.

[6] Feki, M. (2003) "An adaptive chaos synchronization scheme applied to secure communication", Chaos, Solitons and Fractals, Vol. 18, pp 141-148. 
International Journal of Computer Science \& Information Technology (IJCSIT) Vol 3, No 4, August 2011

[7] Murali, K. \& Lakshmanan, M. (1998) "Secure communication using a compound signal from generalized synchronizable chaotic systems", Phys. Rev. Lett. A, Vol. 241, pp 303-310.

[8] Ott, E., Grebogi, C. \& Yorke, J.A. (1990) “Controlling chaos”, Phys. Rev. Lett., Vol. 64, pp 1196-1199.

[9] Ho, M.C. \& Hung, Y.C. (2002) "Synchronization of two different chaotic systems by using generalized active control”, Physics Letters A, Vol. 301, pp 424-428.

[10] Chen, H.K. (2005) "Global chaos synchronization of new chaotic systems via nonlinear control”, Chaos, Solitons \& Fractals, Vol. 23, pp 1245-1251.

[11] Sundarapandian, V. (2011) "Global chaos synchronization of four-scroll and four-wing chaotic attractors by active nonlinear control," International Journal on Computer Science and Engineering, Vol. 3, No. 5, pp. 2145-2155.

[12] Sundarapandian, V. (2011) "Anti-synchronization of Arneodo and Coullet systems by active nonlinear control," International Journal of Control Theory and Applications, Vol. 4, No. 1, pp 25-36.

[13] Liao, T.L. \& Tsai, S.H. (2000) "Adaptive synchronization of chaotic systems and its applications to secure communications”, Chaos, Solitons and Fractals, Vol. 11, pp 1387-1396.

[14] Sundarapandian, V. (2011) "Adaptive control and synchronization of hyperchaotic Cai system", International Journal of Control Theory and Computer Modelling, Vol. 1, No. 1, pp 1-13.

[15] Sundarapandian, V. (2011) "Adaptive synchronization of hyperchaotic Lorenz and hyperchaotic Liu systems", International Journal of Instrumentation and Control Systems, Vol. 1, No. 1, pp $1-18$.

[16] Yu, Y.G. \& Zhang, S.C. (2006) "Adaptive backstepping synchronization of uncertain chaotic systems”, Chaos, Solitons and Fractals, Vol. 27, pp 1369-1375.

[17] Yang, T. \& Chua, L.O. (1999) "Control of chaos using sampled-data feedback control”, Internat. J. Bifurcat. Chaos, Vol. 9, pp 215-219.

[18] Sundarapandian, V. (2011) "Global chaos synchronization of four-wing chaotic systems by sliding mode control”, International Journal of Control Theory and Computer Modelling, Vol. 1 , No. 1, pp 15-31.

[19] Sundarapandian, V. (2011) "Global chaos synchronization of Pehlivan systems by sliding mode control”, International Journal on Computer Science and Engineering, Vol. 3, No. 5, pp 21632169.

[20] Sundarapandian, V. (2011) "Sliding mode controller design for the synchronization of ShimizuMorioka chaotic systems", International Journal of Information Sciences and Techniques, Vol. 1, No. 1, pp 20-29.

[21] Li, G.H. (2005) "Synchronization and anti-synchronization of Colpitts oscillators using active control," Chaos, Solitons \& Fractals, Vol. 26, pp 87-93.

[22] Hu, J. (2005) “Adaptive control for anti-synchronization of Chua's chaotic system”, Physics Letters A, Vol. 339, pp 455-460.

[23] Li, X.F., Chlouverakis, K.E. \& Xu, D.L. (2009) "Nonlinear dynamics and circuit realization of a new chaotic flow: A variant of Lorenz, Chen and Lü”, Nonlinear Analysis, Vol. 10, pp 23572368 .

[24] Tigan, G. \& Opris, D. (2008) “Analysis of a 3-D chaotic system," Chaos, Solitons \& Fractals, Vol. 36, pp 1315-1319.

[25] Hahn, W. (1967) The Stability of Motion, Springer, New York. 


\section{Authors}

Dr. V. Sundarapandian obtained his Doctor of Science degree in Electrical and Systems Engineering from Washington University, Saint Louis, USA under the guidance of Late Dr. Christopher I. Byrnes (Dean, School of Engineering and Applied Science) in 1996. He is currently Professor in the Research and Development Centre at Vel Tech Dr. RR \& Dr. SR Technical University, Chennai, Tamil Nadu, India. He has published over 160 refereed international publications. He has published over 90 papers in National Conferences and over 50 papers in International Conferences. He is the Editor-in-Chief of International Journal of Mathematics and Scientific Computing, International Journal of Instrumentation and Control Systems, International Journal of Control Systems and Computer Modelling, etc. His research interests are Linear and Nonlinear Control Systems, Chaos Theory and Control, Soft Computing, Optimal Control, Process Control, Operations Research, Mathematical Modelling, Scientific Computing using MATLAB etc. He has delivered several Key Note Lectures on Linear and Nonlinear Control Systems, Chaos Theory and Control, Scientific Computing using MATLAB/SCILAB, etc.

Mr. R. Karthikeyan obtained his M.Tech degree in Embedded Systems Technologies from Vinayaka Missions University, Tamil Nadu, India in 2007. He earned his B.E. degree in Electronics and Communication Engineering from Univeristy of Madras, Tamil Nadu, in 2005. He has published over 10 papers in refereed International Journals. He has published several papers on Embedded Systems in National and International Conferences. His current research interests are Embedded Systems, Robotics, Communications and Control Systems.
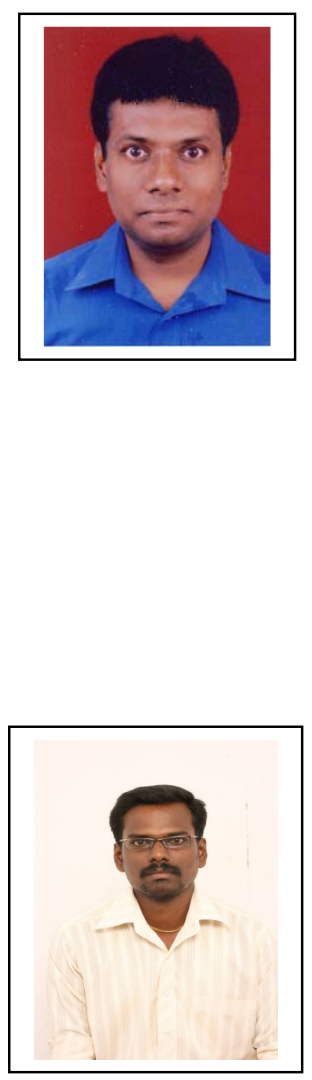